\title{
ON THE RESTRICT-INDUCE MAP OF GROUP CHARACTERS
}

\author{
D. L. WINTER
}

The purpose of this note is to give a new proof of the following theorem due to E. Artin.

THEOREM. Each ordinary irreducible character of a finite group is a linear combination with rational coefficients of characters induced from linear characters of cyclic subgroups.

Let $G$ be a finite group and let $K_{1}, \cdots, K_{n}$ be the classes of conjugate elements of $G$. Then the ordinary irreducible characters $\chi_{1}$, $\cdots, \chi_{n}$ form a basis of the vector space $U$ of all complex-valued class functions on $G$ over the complex number field. This basis is orthonormal relative to the usual inner product. There is another orthonormal basis $\alpha_{1}, \cdots, \alpha_{n}$ defined by

$$
\begin{aligned}
\alpha_{i}(g) & =0 \quad \text { if } g \in G-K_{i}, \\
& =|C(g)|^{1 / 2} \quad \text { if } g \in K_{i},
\end{aligned}
$$

where $|C(g)|$ is the order of the centralizer $C(g)$ of $g$ in $G$.

Let $H$ be a subgroup of $G$. We define a linear transformation $T$ of $U$ by $T(\theta)=(\theta \mid H)^{*}$, the class function on $G$ obtained by inducing the restriction of $\theta$ to $H$. It will be noted that $T$ is symmetric, for, applying Frobenius reciprocity twice, we have

Let

$$
\begin{aligned}
(T(\theta), \eta)_{G} & =\left((\theta \mid H)^{*}, \eta\right)=(\theta|H, \eta| H)_{H} \\
& =\left(\theta,(\eta \mid H)^{*}\right)=(\theta, T(\eta))_{G} .
\end{aligned}
$$

$$
T\left(\chi_{i}\right)=\sum_{j} a_{i j} \chi_{j}
$$

so that $A=\left(a_{i j}\right)$ is the matrix of $T$ relative to the basis $\chi_{1}, \cdots, \chi_{n}$. It is known that each $a_{i j}$ is a nonnegative rational integer.

If $K_{i} \cap H$ is empty, the formula for the value of an induced character implies $T\left(\alpha_{i}\right)=0$. If $K_{i} \cap H$ is nonempty, then

$$
K_{i} \cap H=C_{1}+\cdots+C_{s}
$$

where each $C_{j}$ is a class of $H$. Let $g \in K_{i}$ and let $g_{j} \in C_{j, s} j=1, \cdots, s$. Then

Received by the editors December 16, 1966. 


$$
\begin{aligned}
T\left(\alpha_{i}\right)(g) & =\left[|C(g)|\left|K_{i} \cap H\right| /|H|\right] \alpha_{i}(g) \\
& =\left[(|C(g)| /|H|) \sum_{j}\left|H: H \cap C\left(g_{j}\right)\right|\right] \alpha_{i}(g) \\
& =\left[\sum_{j}|C(g)| /\left|H \cap C\left(g_{j}\right)\right|\right] \alpha_{i}(g) \\
& =\left[\sum_{j}\left|C\left(g_{j}\right): C\left(g_{j}\right) \cap H\right|\right] \alpha_{i}(g) .
\end{aligned}
$$

Since $T\left(\alpha_{i}\right)(g)=\alpha_{i}(g)=0$ for $g \in G-K_{i}$, we have

$$
T\left(\alpha_{i}\right)=r_{i} \alpha_{i}
$$

where $r_{i}=\sum_{j}\left|C\left(g_{j}\right): C\left(g_{j}\right) \cap H\right|$ is a sum of positive integers. Hence $\alpha_{1}, \cdots, \alpha_{n}$ is a basis of eigenvectors of $T$ and the eigenvalues $r_{1}, \cdots, r_{n}$ are nonnegative integers. The above shows that the rank of $T$ is the number of classes of $G$ meeting $H$. The kernel of $T$ is the set of class functions on $G$ vanishing on those classes meeting $H$.

The two matrices of $T, A$ and diag $\left\{r_{1}, \cdots, r_{n}\right\}$, are similar over the complex number field. Since their entries are rational numbers, they are already similar over the rational number field $Q$. This implies the existence of a basis $\theta_{1}, \cdots, \theta_{n}$ of eigenvectors of $T$ which are $Q$-linear combinations of $\chi_{1}, \cdots, \chi_{n}$ with eigenvalues $r_{1}, \cdots, r_{n}$.

We consider $\theta_{1}, \cdots, \theta_{n}$ as a basis of the vector space $V(G)$ of all $Q$-linear combinations of $\chi_{1}, \cdots, \chi_{n}$ and restrict $T$ to $V(G)$. Assume the notation is chosen so that $r_{i} \neq 0, i=1, \cdots, t$ while $r_{i}=0$ for $i=t+1, \cdots, n$. Note that for $i \leqq t, \theta_{i}(g)=0$ if $g$ lies in a class of $G$ not meeting $H$, because $\theta_{i}(g)=\left(1 / r_{i}\right) T\left(\theta_{i}\right)(g)=0$. On the other hand for $i>t, \theta_{i}(g)=0$ if $g$ lies in a class meeting $H$. Now let $\chi$ be an irreducible character of $G$ and $a_{1}, \cdots, a_{n}$ rational numbers such that $\chi=\sum_{j=1}^{n} a_{j} \theta_{j}$. Set $\mu=\sum_{j=1}^{t} a_{j} \theta_{j}$. It follows that $\mu$ vanishes on those classes not meeting $H$ and that $\mu|H=\chi| H$. Furthermore,

$$
\mu=\sum_{j=1}^{t} \frac{a_{j}}{r_{j}}\left(r_{j} \theta_{j}\right)=\sum_{j=1}^{t} \frac{a_{j}}{r_{j}}\left(\theta_{j} \mid H\right)^{*} .
$$

Since $\theta_{j} \mid H$ is a $Q$-linear combination of irreducible characters of $H$, we have the following proposition.

Proposition. Given an irreducible character $\chi$ of $G$ and a subgroup $H$ of $G$, there exists a Q-linear combination $\mu$ of characters of $G$ induced from irreducible characters of $H$ such that $\mu=0$ on classes of $G$ not meeting $H$ and $\mu|H=\chi| H$. 
The function $\mu$ is not uniquely determined. In the following paragraph we write $\chi(H)$ for one such function, indicating its dependence on the given character $\chi$ and the given subgroup $H$. The particular choice of $\chi(H)$ is immaterial.

Now let $g_{i} \in K_{i}, i=1,2, \cdots, n$ and let $H_{i}$ be the cyclic subgroup generated by $g_{i}$. Let $\chi$ be an irreducible character of $G$. The proof of the main result is completed by noting

$$
\begin{aligned}
\chi= & \chi\left(H_{1}\right)+\chi\left(H_{2}\right)+\cdots+\chi\left(H_{n}\right) \\
& -\chi\left(H_{1} \cap H_{2}\right)-\chi\left(H_{1} \cap H_{3}\right)-\cdots-\chi\left(H_{n-1} \cap H_{n}\right) \\
& +\chi\left(H_{1} \cap H_{2} \cap H_{3}\right)+\cdots+\chi\left(H_{n-2} \cap H_{n-1} \cap H_{n}\right) \\
& \vdots \\
& +(-1)^{n+1} \chi\left(H_{1} \cap H_{2} \cap \cdots \cap H_{n}\right)
\end{aligned}
$$

where in the $k$ th row there is one and only one term for each possible intersection of $k$ of the $H_{i}$ 's. (This expression holds whenever $H_{1}$, $\cdots, H_{n}$ is a set of subgroups of $G$ such that each class of $G$ meets at least one $H_{i}$.)

Michigan State University 